\title{
Set-Portfolio Selection with the Use of Market Stochastic Bounds
}

\author{
SERGIO ORTOBELLI LOZZA ${ }^{1}$, ENRICO ANGELELLI ${ }^{2}$, DANIELE TONINELLI $^{1}$, \\ ${ }^{1}$ MSIA Department \\ University of Bergamo \\ 24127 - Via dei Caniana, 2; Bergamo - Italy; \\ e-mail addresses: sol@unibg.it; daniele.toninelli@unibg.it \\ ${ }^{2}$ Quantitative Methods Department \\ University of Brescia \\ 25122 - C.da S. Chiara, 50; Brescia - Italy; \\ e-mail address: angele@eco.unibs.it
}

\begin{abstract}
This paper proposes an ex-post comparison of portfolio selection strategies. These are applied to certain preselected assets among about ten thousand stocks on the global market. In particular, we preselected a few assets for each portfolio selection problem, taking into account different return characteristics. The preselecting criteria take into account the joint Markovian behavior of the returns; furthermore, they consider the assets who optimize the association with market stochastic bounds, having the highest ex-ante reward-risk performance. The results obtained with different pre-selection criteria are merged in order to identify assets with common characteristics which are appealing for investors. The impact of assets pre-selection on the portfolio choices is also studied. In particular, we compare the performance of different strategies that use or do not use the preselecting criteria. We finally propose the comparison of the ex-post final wealth obtained with the optimization of several reward-risk functionals that use the stochastic bounds of the preselected assets. For every comparison, we assume that the returns follow a non-parametric Markov chain, where the investors recalibrate their portfolios on a weekly basis.
\end{abstract}

Key-Words: Markov chains, stochastic bounds, portfolio strategies, computational complexity.

\section{Introduction}

The purpose of this paper is twofold: we evaluate the effects of a sophisticated asset pre-selection methodology and we propose certain portfolio strategies that use market stochastic bounds. We model the return portfolios with a Markov chain. Under this distributional hypothesis, we preselect up to 30 assets among about ten thousand stocks on the global market. The selection is limited to 30 assets only, because we want to emphasize the pre-selection effects. The introduced strategies also allow us to explain the very high returns that could not be explained with the efficient market hypothesis (see Fama, 1970). Moreover, we evaluate the impact of using different types of distances between optimal portfolio and market stochastic bounds. Therefore, we compare the ex-post performances of certain proper portfolio strategies.

In this work, we take into consideration a portfolio selection procedure divided into three phases. The paper focuses in particular on the study of the first and last phases. In the first phase, we preselect a few assets that satisfy certain performance criteria. Moreover, we define "good" assets considering performance ratios based on a Markovian evolution of the assets (see Angelelli and Ortobelli, 2009a, 2009b) or simply assuming equi-distributed historical observations. In the second phase of the portfolio selection, the dimensionality of the preselected assets is reduced, identifying a few common factors to approximate the asset returns. In particular, the principal component analysis ( $P C A)$ is applied to the Pearson correlation matrix. This matrix is computed on the Markovian forecast of future returns. Thus, the returns are approximated by regressing on the first few components obtained with the PCA. This second phase of the analysis is discussed with more detail in Angelelli and Ortobelli (2010). In the third and last phase, we apply the model to real data concerning 14 countries. In particular, we first evaluate the impact of the pre-selection criteria by comparing the performances of different strategies based, or not 
based, on such preselecting criteria. Secondly, we assess certain portfolio selection strategies based on the optimization of functionals of future wealth and of market stochastic bounds. The portfolio strategies dealt with in the paper are based on an estimate of the returns distribution at future times; thus, we found a substantial difference between portfolio selection strategies developed using their Markovian evolution compared to strategies based on the assumption of equi-distribution of return historical observations. To deal with the complexity of portfolio selection problems based on the Markovianity of the gross returns, we use an heuristic for global optimization (see Angelelli and Ortobelli, 2009a).

The paper is organized as follows. In Section 2, we discuss the first phase of the portfolio selection procedure, illustrating how to model a non-parametric Markov chain and formalizing the pre-selection criteria. In Section 3 and 4, we discuss the third phase. In particular, Section 3 shows the impact of portfolio pre-selection on the U.S. stock market; Section 4 compares different strategies that use market stochastic bounds on the global market. The last Section briefly summarizes the paper.

\section{Pre-selection criteria with non- parametric Markov processes}

In this section we deal with the portfolio selection problem. Our analysis is applied to $n$ risky assets with gross returns $z_{t+1}=\left[z_{1, t+1}, \ldots, z_{n, t+1}\right]^{\prime}$. We assume that either returns are simply equi-distributed or the portfolio process is described by a homogeneous Markov chain with $N$ states. In this last case, approximation of future behavior is based on the implicit assumption that all portfolios of returns follow a Markov chain defined on a filtered probability space $\left(\Omega, \mathfrak{I},\left(\mathfrak{I}_{t}\right)_{0 \leq t \leq \infty}, P\right)$. In this context filtration is fundamental, since the stopping times of the filtration are used in the pre-selection framework. The Markovian hypothesis serves to forecast future behavior of wealth. All of the analyses in the dynamic framework are performed based on forecasted wealth. The vector of the positions taken in the $n$ risky assets is denoted by $x=\left[x_{1}, \ldots, x_{n}\right]^{\prime}$ and the portfolio return during the period $[t, t+1]$ is given by $z_{(x), t+1}=x^{\prime} z_{t+1}=\sum_{i=1}^{n} x_{i} z_{i, t+1}$. In particular, we assume that investors want to maximize the performance of their choices at a given future date $T$.

The classic portfolio selection problem, in which no short sales are allowed, can be represented as the maximization of a given performance applied to the random portfolio of gross returns $z_{(x), t+1}$. Since no short sales are allowed, the portfolio of weights belongs to the (n-1)-dimensional simplex $S=\left\{x \in \mathrm{R}^{n} \mid \sum_{i=1}^{n} x_{i}=1 ; x_{i} \geq 0\right\}$. In particular, we suggest selecting those assets that satisfy some optimality criteria, considering several properties of wealth behavior. The factors we should consider as preselecting criteria are:

1) Timing (i.e., we consider that investors want to maximize the time it takes for wealth to reach a given lower bound and minimize the time it takes for wealth to reach a given upper bound).

2) Markovian behavior of wealth.

3) Asymptotic behavior of wealth.

4) The consistency with investors' preferences.

5) The association with market stochastic bounds.

With regards to this last point, we recall that the upper and lower market stochastic bounds among $m$ assets with returns $z_{i}$ are respectively given by the maximum and minimum of the marginal returns (i.e., $\max _{i \leq m} z_{i}$ and $\min _{i \leq m} z_{i}$ ) when no short sales are allowed (for further details, see Ortobelli and Tichy, 2010). In fact:

$$
\min _{i \leq m} z_{i} \leq z_{(x), t+1} \leq \max _{i \leq m} z_{i}
$$

for any time $t$ and for any vector of portfolio weights $x$ belonging to $S=\left\{x \in \mathrm{R}^{m} \mid \sum_{i=1}^{m} x_{i}=1 ; x_{i} \geq 0\right\}$. As discussed by Ortobelli and Tichy (2009 and 2010), investors want to maximize the association with the upper stochastic bound and minimize the association with the lower bound. Concordance measures are generally used to measure the concordance / dependence / association between random variables (see Nelsen, 2006). We recall that two random variables (e.g., $X, Y$ ) with independent replications, $\left(X_{1}, Y_{1}\right)$ and $\left(X_{2}, Y_{2}\right)$, are concordant if $X_{1}<X_{2}\left(X_{1}>X_{2}\right)$ implies $Y_{1}<Y_{2}\left(Y_{1}>Y_{2}\right)$. Similarly, two variables are discordant if $X_{1}<X_{2}\left(X_{1}>X_{2}\right)$ implies $Y_{1}>Y_{2}\left(Y_{1}<Y_{2}\right)$. Gini and Kendall coefficients are traditional concordance measures. Clearly, investors want:

1) their portfolios to be as much as possible concordant with the upper stochastic bound, since they want to increase their wealth;

2) their portfolios to be as much as possible discordant with the lower stochastic bound, since they want to reduce their losses.

Therefore, we take into account these investor preferences to select a limited number of assets. 
The approach to future wealth can generally be considered static or dynamic:

i) with the static approach, we assume that historical observations are equally distributed. Moreover, the investor maximizes a functional of future wealth that is independent from his temporal horizon $[0, T]$, i.e. he solves problems of the following type:

$$
\max _{x \in S} f\left(z_{(x), t+1}\right)
$$

ii) With the dynamic approach, we consider that gross returns follow a Markov chain. Therefore, the investor maximizes a functional of future wealth, that should be determined under this hypothesis, i.e. he solves problems of the following type:

$$
\max _{x \in S} f\left(W_{T}\left(z_{(x)}\right)\right),
$$

where $W_{T}\left(z_{(x)}\right)$ is the predicted wealth obtained after $T$ working days of investing in the portfolio $z_{(x)}=x^{\prime} z$.

Starting from the static approach (i), we represent the classic myopic approach, which does not use a time evolution of the wealth process. In the dynamic context (ii), we consider instead all the admissible wealth Markovian processes $W\left(z_{(x)}\right)=\left\{W_{t}\left(z_{(x)}\right)\right\}_{t \geq 0}$. They are defined on the filtered probability space that depends on an initial portfolio of weights $x \in S$.

In the pre-selection criteria of our work, we consider both approaches. We select certain assets assuming approach (i) and other assets considering approach (ii). Then, the union of all these assets is used for portfolio selection.

In order to select a few desirable assets, both selection approaches are based on three steps:

- Step 1: a desirable ordering criterion is taken into account.

- Step 2: the assets are ordered by this ordering criterion.

- Step 3: the 20 best assets that satisfy the dynamic approach criteria and the 10 best assets that satisfy the static approach criteria are selected.

We decided to use a maximum of 30 preselected assets for two main reasons: to emphasize the impact of pre-selection, and to take into account the point of view of a "small" investor. The first point will be explained in Section 3, where we show that with a few preselected assets we obtain much more wealth than with the full diversified portfolio. On the other hand, we refer to the paradigm about diversification, showing that even by using a few preselected assets we were able to reduce the investor's risk during a period of crisis.

In order to deal with the pre-selection methodology, we first argue how to approximate the Markovian evolution of wealth (Section 2.1), then we discuss three ordering criteria with the static approach (Section 2.2) and five criteria with the dynamic approach (Section 2.3).

\subsection{The Markovian evolution process}

In order to consider the Markovian behavior of wealth, we assume that each portfolio follows a Markov chain.

Let $\left(\min _{k} z_{(x), k} ; \max _{k} z_{(x), k}\right)$ be the range of the portfolio gross returns, where $z_{(x), k}$ is the $k$-th past observation of the portfolio $z_{(x)}$. Without a loss of generality, we assume that the $N$ states $z_{(x)}^{(i)}$ of portfolio gross return are ordered as follows: $z_{(x)}^{(i)}>z_{(x)}^{(i+1)}$ for $i=1, \ldots, N-1$. Since we want to obtain a recombining tree of the Markov chain, we first divide the portfolio support $\left(\min _{k} z_{(x), k} ; \max _{k} z_{(x), k}\right)$ in $N\left(a_{(x), i} ; a_{(x), i-1}\right)$ intervals, where:

$$
a_{(x), i}=\left(\frac{\min _{k} z_{(x), k}}{\max _{k} z_{(x), k}}\right)^{i / N} \cdot \max _{k} z_{(x), k}, \quad i=0,1, \ldots, N
$$

This measure is decreasing with index $i$. We then compute the return associated with each state as the geometric average of the extremes of the interval $\left(a_{(x), i} ; a_{(x), i-1}\right)$, that is:

$$
z_{(x)}^{(i)}=\sqrt{a_{(x), i} a_{(x), i-1}}=\max _{k} z_{(x), k}\left(\frac{\max z_{(x), k}}{\min z_{(x), k}}\right)^{\left(\frac{1-2 i}{2 N}\right)},
$$

where: $i=1,2, \ldots, N$.

Consequently: $z_{(x)}^{(i)}=z_{(x)}^{(1)} u^{1-i}$, where:

$$
u=\left(\frac{\max z_{(x), k}}{\min z_{(x), k}}\right)^{1 / N}>1
$$

Let us assume that the initial wealth $W_{0}$ at time 0 is equal to 1 , while for each possible wealth $W_{t}$ at time $t$ we have $N$ possible different values $W_{t+1}=W_{t} z_{(x)}^{(i)}$ at time $t+1(i=1,2, \ldots, N)$. Seeing the recombining effect of the Markov chain, we have $1+k(N-1)$ possible values after $k$ steps of wealth $W_{k}\left(z_{(x)}\right)$. They are given by $w_{(x)}^{(i, k)}=\left(z_{(x)}^{(1)}\right)^{k} u^{(1-i)}(i=1, \ldots,(N-1) k+1)$, where the $i$-th node at time $k$ of the Markovian tree 
corresponds to wealth $w_{(x)}^{(i, k)}$. Moreover, all possible values of random wealth, $W_{k}\left(z_{(x)}\right)$, can be stored in a matrix with $k$ columns and $1+k(N-1)$ rows resulting in $O\left(N k^{2}\right)$ memory space requirement. Since we assume a homogeneous Markov chain, the transition matrix $P=\left[p_{i, j}\right]$ does not depend on time and the entries $p_{i, j}$ are estimated using the maximum likelihood estimates $\hat{p}_{i, j}=\frac{\pi_{i j}(K)}{\pi_{i}(K)}$, where $\pi_{i j}(K)$ is the number of observations (out of $K$ observations) that transit from the $i$-th state to the $j$-th state and $\pi_{i}(K)$ is the number of observations (out of $K$ observations) in the $i$-th state (for the statistical properties of these estimators, see D'Amico, 2003). Following the idea of Iaquinta and Ortobelli (2006), we can compute the distribution function of future gross returns. In particular, the $(N-1) k+1$ dimensional vector $p^{(k)}$ (representing the unconditional distribution at a given time $k(k=0,1$, $2, \ldots, T)$ of wealth $\left.W_{k}\left(z_{(x)}\right)\right)$ can be computed by means of a sequence of matrixes $\left\{Q^{(k)}\right\}_{k=0,1, \ldots, T}$, where $Q^{(k)}=\left[q_{i, j}^{(k)}\right]_{\substack{1 \leq i \leq(N-1) k+1 \\ 1 \leq j \leq N}}$ and $q_{i, j}^{(k)}$ is the unconditional probability to obtain wealth $w_{(x)}^{(i, k)}$ and to be in the state $z_{(x)}^{(j)}$ at time $k$. In particular, $Q^{(0)}=\left[p_{1}, \ldots, p_{N}\right]$, where $p_{i}$ is the unconditional probability to be in the $i$-th state at time 0 . Thus, $p^{(0)}=1=Q^{(0)} \cdot \mathbf{1}_{N}$, where $\mathbf{1}_{N}$ is the unity vector column. In general, for $k=1,2, \ldots, T$, the vector $p^{(k)}$ is given by $p^{(k)}=Q^{(k)} \cdot \mathbf{1}_{N}$, where $Q^{(k)}$ is recursively defined as $Q^{(k)}=\operatorname{diagM}\left(Q^{(k-1)} \cdot P\right)$. diagM is a linear operator defined for any $m, n \in N$ as diagM: $R^{m n} \rightarrow R^{(m+n-1) n}$. It is associated with any $m \times n$ matrix $A=\left[a_{i j}\right]$ the $(m+n-1) \times n$ matrix obtained by simply shifting down the $j$-th column by $(j-1)$ rows (for further details see Iaquinta and Ortobelli, 2006, and Angelelli and Ortobelli, 2009a).

Matrix $Q^{(k)}$ is called unconditional evolution matrix of the Markov chain or, simply, evolution matrix. The algorithm used to compute probabilities has a computational complexity of $O\left(N^{3} k^{2}\right)$. As shown in Angelelli and Ortobelli (2010), future wealth $W_{T}\left(z_{(x)}\right)$ is generally better approximated by a stable
non-Gaussian law. This suggests that markets are not as efficient as the efficiency hypothesis states.

\subsubsection{Consequences of the Markovian hypothesis}

When we assume that the portfolios follow a Markov chain, we can distinguish different types of possible strategies.

According to the definition given in Angelelli and Ortobelli (2009b), we call OA expected utility the above functional $E\left(u\left(W_{T}\left(z_{(x)}\right)\right)\right)$ when it is computed under the assumption that the gross return of each portfolio follows a Markov chain with $N$ states. The OA expected utility is given by:

$$
\begin{aligned}
E\left(u\left(W_{T}\left(z_{(x)}\right)\right)\right) & =u\left(\hat{W}_{T}\left(z_{(x)}\right)\right) \cdot Q^{(T)} \cdot \mathbf{1}_{N}= \\
& =u\left(\hat{W}\left(z_{(x)}\right)\right) \cdot p^{(T)},
\end{aligned}
$$

where $\quad \hat{W}_{T}\left(z_{(x)}\right)=\left[w_{(x)}^{(1, T)}, \ldots, w_{(x)}^{((N-1) T+1, T)}\right]$ is the $(N-1) T+1$ dimensional vector of the final wealth and $u\left(\hat{W}_{T}\left(z_{(x)}\right)\right)=\left[u\left(w_{(x)}^{(1, T)}\right), \ldots, u\left(w_{(x)}^{((N-1) T+1, T)}\right)\right]$ is the utility evaluated on the final wealth. Formula 10 is a logical consequence of the methodology that describes the Markovian tree. As a matter of fact, $p^{(T)}=Q^{(T)} \cdot \mathbf{1}_{N}$ gives the distribution of the final wealth.

Angelelli and Ortobelli (2009b) have shown that standard optimization algorithms are not adequately suited to solve the global optimization problem of OA expected utility. Thus, we use the same optimization heuristic proposed by Angelelli and Ortobelli (2009a) to solve portfolio optimization problems. Starting from an initial feasible portfolio solution, $\bar{x}$, the heuristic algorithm tries to iteratively update the current solution with a better one. Improving solutions, if any, are searched on a predefined grid of points fixed on the directions $x-e_{i}(i=1,2, \ldots, n)$, where $x$ is the current portfolio and $e_{i}$ is the portfolio where the share of asset $i$ is equal to 1 and all other assets have share equal to 0 . If a better solution is found on a search direction, the current solution is updated and the search continues from the new one. If none of the directions provides an improved solution, the search ends. The main advantages of this algorithm are the following (for more details, see Angelelli and Ortobelli, 2009a):

1) The algorithm allows the global optimum to be approximated with a given error, when the optimum is unique.

2) The algorithm allows the whole simplex to be 
explored.

3) The computational complexity is much less than that of classic algorithms for global optimum such as Simulated Annealing type algorithms (see Leccadito et al., 2007, and the references therein).

\subsection{Pre-selection criteria (static approach)}

With the pre-selection criteria of the static approach we only take into account the consistency of investors' preferences and the association with the market stochastic bounds. In particular, we order the assets considering the following three criteria:

1) wealth obtained in the last 120 working days, i.e., the assets are ordered with respect to the ratio:

$$
\frac{P_{t}}{P_{t-120}}
$$

where $P_{t}$ and $P_{t-120}$ are, respectively, the adjusted prices at time $t$ and $t-120$ (where 120 working days are about six months of data);

2) the Sharpe ratio:

$$
\frac{E\left(z_{i}-z_{f}\right)}{\operatorname{St} \cdot \operatorname{dev}\left(z_{i}\right)},
$$

where the mean and the standard deviation of the $i$-th asset are approximated by the empirical mean and standard deviation of the last 120 working days (we assume that the riskless is null, that is $\left.z_{f}=1\right)$ :

$$
E\left(z_{i}\right) \cong \frac{1}{120} \sum_{t=1}^{120} z_{i, t},
$$

and:

$$
\operatorname{St.dev}\left(z_{i}\right) \cong\left(\frac{1}{120} \sum_{t=1}^{120}\left(z_{i, t}-E\left(z_{i}\right)\right)^{2}\right)^{0.5} .
$$

3) the Gini performance ratio:

$$
\frac{\gamma\left(z_{i}, \max _{i \leq m} z_{i}\right)}{1.1+\gamma\left(z_{i}, \min _{i \leq m} z_{i}\right)} ;
$$

this ratio is based on the Gini $\gamma$ concordance measure; the sample estimation of this measure is given by:

$$
\gamma(X, Y)=\frac{1}{\left[\frac{n^{2}}{2}\right]} \sum_{i=1}^{n}\left|p_{i}-q_{i}-n-1\right|-\left|p_{1}-q_{1}\right|,
$$

where $n=120$ is the number of observations, $p_{i}$ and $q_{i}$ are, respectively, the ranks of the random variables $X$ and $Y$.

The first two criteria are consistent with choices of non-satiable investors. While maximizing the Gini performance ratio, we maximize the concordance between the portfolio and the upper stochastic bound and we minimize the concordance between the portfolio and the lower stochastic bound.

Using these three criteria, we preselect ten desirable assets for nonsatiable investors. In particular, the choice includes the first ten assets, among $m$, with the best common performance measures (Sharpe ratio, wealth obtained in the last six months, and Gini performance ratio).

\subsection{Pre-selection criteria (dynamic approach)}

With the pre-selection criteria of the dynamic approach we take into account the consistency with investors' preferences, the timing of the choices, the association with market stochastic bounds, and the Markovian and asymptotic behavior of wealth. In particular, we assume that each portfolio of returns follows a Markov chain. Then, we preselect assets considering the following five ordering criteria:

1) The expected power utility:

$$
E\left(u\left(W_{T}\left(z_{i}\right)\right)\right) ;
$$

this is the predicted wealth $W_{T}\left(z_{i}\right)$ obtained after $T=20$ working days investing in the $i$-th asset (for any $i=1,2, \ldots, n$ ). In formula (2) $u(W)=\frac{W^{g}}{g}$, with $g=0.9$.

2) The Sharpe ratio:

$$
\frac{E\left(W_{T}\left(z_{i}\right)\right)-1}{\text { St.dev. }\left(W_{T}\left(z_{i}\right)\right)},
$$

where $W_{T}\left(z_{i}\right)$ is the predicted wealth obtained after $T=20$ working days investing in the $i$-th asset (for any $i=1,2, \ldots, n$ ).

3) The Pearson performance ratio, based on the Pearson linear correlation $(\tau)$ :

$$
\frac{\tau\left(W_{T}\left(z_{i}\right), W_{T}\left(\max _{i \leq m} z_{i}\right)\right)}{1.1+\tau\left(W_{T}\left(z_{i}\right), W_{T}\left(\min _{i \leq m} z_{i}\right)\right)} .
$$

4) The timing ratio:

$$
\frac{E\left(\pi_{1}\right)}{E\left(\pi_{2}\right)},
$$

where $\pi_{1}$ and $\pi_{2}$ are two stopping times of the filtration defined as:

$$
\pi_{1}\left(z_{i}\right)=\min \left(T, \inf \left\{k \in[0, T] \mid W_{k}\left(z_{i}\right) \leq 0.95\right\}\right)
$$

and

$$
\pi_{2}\left(z_{i}\right)=\min \left(T, \inf \left\{k \in[0, T] \mid W_{k}\left(z_{i}\right) \geq 1.2\right\}\right) .
$$


These stopping times give the first time (belonging to $[0, T]$ ) that wealth produced by the $i$-th asset reaches, respectively, the bounds 0.95 and 1.2 .

5) The OA-stable ratio:

$$
\frac{\delta_{W_{T}\left(z_{i}\right)}}{\operatorname{ETL}_{\beta}\left(W_{T}\left(z_{i}\right)-E\left(W_{T}\left(z_{i}\right)\right)\right)},
$$

where $\delta_{W_{T}\left(z_{i}\right)}$ is the location parameter of the best quantile, stable Paretian approximation of the wealth $W_{T}\left(z_{i}\right)$, the denominator $E T L_{\beta}$ (expected tail loss or average value at risk for $\beta=0.05$ ) is computed under this distributional assumption using the Stoyanov et al. algorithm (2006).

Therefore, we choose the first 20 assets (among $m$ ) with the best common performance measures of predicted wealth: expected power utility, Sharpe, association type ratio, timing ratio and stable ratio. Together with the 10 assets selected with the static approach, the total of pre-selected desirable assets is between 20 and 30. As a matter of fact, some assets could be selected with both approaches (static and dynamic).

\section{Impact of pre-selection criteria}

In this section, we evaluate the impact of pre-selection criteria. In our analysis, we considered all the equities active during the last six months (120 working days) on the following 14 markets: Hong Kong, Shanghai, Singapore, Taiwan, Tokyo Stock Exchange, Nasdaq, NYSE, Frankfurt, Milan, London, Euronext Amsterdam, Euronext Paris, Euronext Lisbon, Euronext Brussels. The data source is DATASTREAM. We recalibrated the portfolio every month ( $T=20$ working days) to be consistent with dynamic pre-selection analysis. At each recalibration time, there are about ten thousand active assets that can be selected for the portfolio. The number of assets changes at each recalibration time, since market size, vacations and rules are different for each market. For the evaluation, we only consider observations for the last six months, as they are supposed to have the highest impact in future choices (as proved by several experiments). In order to evaluate the impact of preselection, we ex-post compared the wealth sample paths of the following three possible strategies.

1) The first strategy considers, at each recalibration time, all the active assets in the entire group of markets. This strategy implies maximum diversification: we invest $1 / m$ in each equity, where $m$ is the number of the assets.

2) In the second strategy, pre-selection criteria are applied in order to consider the preselected assets only. The number of preselected assets is usually equal to 30 , since the two pre-selection plans (static and dynamic) almost never select common assets. As parameters of the Markov chain, we use $N=9$ states and the temporal horizon $T=20$. Angelelli and Ortobelli 2009a have shown that by using $N=9$ states and $T=20$, it is possible to obtain a good approximation of the final wealth distribution. Even in this case we consider maximum diversification on the preselected assets and we invest $1 / k$ (where $k$ is the number of preselected assets) in each preselected asset.

3) Within the third strategy, the best preselected assets are still used. On these assets we maximize the classical Sharpe Ratio $\left(\frac{E\left(x^{\prime} z\right)-1}{\operatorname{St} \operatorname{dev}\left(x^{\prime} z\right)}\right)$ at each recalibration time during the last two years.

In the ex-post analysis we do not consider transaction costs. We assume to start with an initial wealth $W_{0}=1$, then we define the optimal portfolio with each strategy and at each recalibration time. The period considered for the ex-post analysis goes from August 15, 2007 to July 3, 2010. Lastly, we compute the ex-post wealth for each optimal choice, i.e.:

$$
W_{t}=W_{t-20} * z^{e x-p o s t}
$$

where $z^{\text {ex-post }}$ is the ex-post gross return observed at time $t$ with the choice carried out at time $t-20$. The graph in Figure 1 compares the sample paths of expost final wealth.

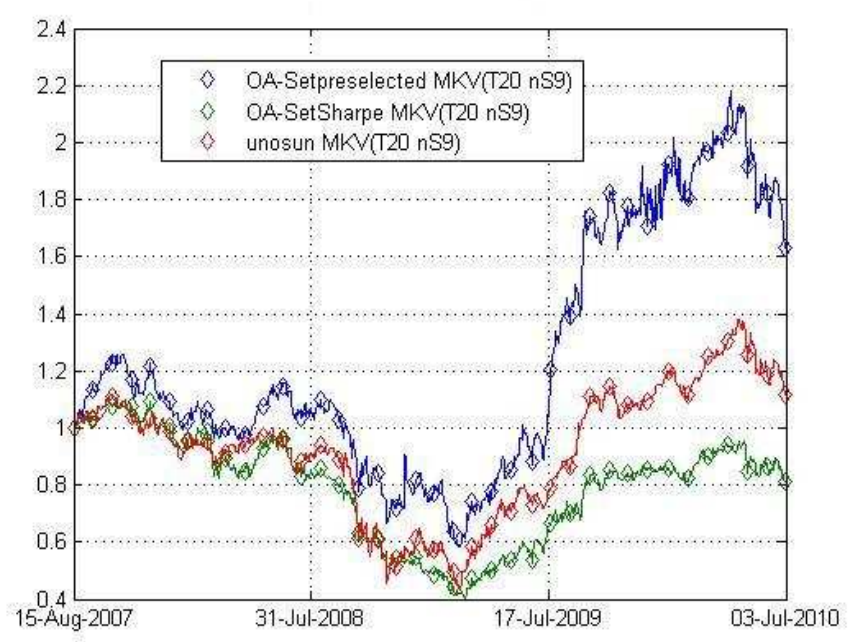

Figure 1. Ex-post final wealth process when pre-selection applies (recalibration every 20 days). 
Figure 1 shows the effect of the crisis during the last 2 years. As a matter of fact, the trend decreases from August 15, 2007 to March 15, 2009 (with a loss of about 40/50\%) and during the months of May and June 2010, due to the countries' risk credit crisis. The blue line shows the ex-post wealth with maximum diversification among 30 preselected assets. The red line shows the ex-post wealth with maximum diversification among all assets (about ten thousand). The green line shows the ex-post wealth when we maximize the Sharpe ratio.

From Figure 1, we deduce that:

1) Diversification has a very strong impact on portfolio selection;

2) Pre-selection has a very important impact on portfolio choices.

A surprising result is given by the maximum diversification strategy which, alone, gives about $10 \%$ more of wealth in two years. However, maximum diversification among all assets does not consider transaction costs, which should considerably affect this strategy. The best performance is given when we consider pre-selection and diversification together. This increases wealth by more than $60 \%$, during the two years of the crisis. The effect of diversification is amplified if we consider that the worst performance is obtained by applying the classic Sharpe ratio on preselected assets, since the ambiguity effect is prevalent (see Pflug et al., 2010). As a matter of fact, the ambiguity risk measures the "modelization risk". In practical terms, this suggests that we should not consider a static approach to model the risk. Given the above, in the next section we will analyze the effects of dynamic strategies on preselected assets.

\section{An ex-post comparison among $\mathrm{OA}$ portfolio strategies applied to preselected assets}

In this section, we propose an ex-post comparison among OA strategies that take into account different types of distances from stochastic bounds. As for the previous analysis, we consider all the assets of the above 14 markets. Moreover, we compare the performance of three Markovian strategies applied on assets preselected among the last year's data (from August 14, 2009 to July 16, 2010). In this analysis, we consider $N=9$ states of the Markov chain and a temporal horizon of $T=20$ working days. We recalibrate the portfolios every 20 days to reduce the computational time of the analysis. In the Markovian strategies, we only use observations from the last six months; thus we reduce the randomness of the choice approximating the preselected assets with a principal component analysis (PCA) (for further details about the PCA, see Ortobelli and Tichy 2010). This means that we preselect no more than 30 optimal assets. The PCA analysis is applied to the preselected assets and we take into account the first 20 principal components $\left(f_{i}, i=1, \ldots, 20\right)$. Then the returns are regressed on these factors and the approximated returns are used in portfolio selection problems.

The three strategies are aimed at maximizing the following performance ratios:

1) Absolute distributional distance ratio:

$$
\frac{\int_{-\infty}^{+\infty} \sum_{t=1}^{T}\left|F_{W_{t}\left(z_{(x)}\right)}(u)-F_{W_{t}\left(\min _{i} z_{i}\right)}(u)\right| d u}{\int_{-\infty}^{+\infty} \sum_{t=1}^{T}\left|F_{W_{t}\left(z_{(x)}\right)}(u)-F_{W_{t}\left(\max _{i} z_{i}\right)}(u)\right| d u},
$$

where $F_{W_{t}\left(z_{(x)}\right)}, \quad F_{W_{t}\left(\max _{i} z_{i}\right)}$ and $F_{W_{t}\left(\min _{i} z_{i}\right)}$ are the estimated distributions of wealth at time $t$ obtained, respectively, from the portfolio $z_{(x)}$, the upper $\left(\max _{i} \mathrm{z}_{i}\right)$ and lower $\left(\min _{i} \mathrm{z}_{i}\right)$ stochastic bounds. This ratio minimizes the absolute distributional distance between the upper stochastic bound and the portfolio and maximizes the same distance between the portfolio and the lower stochastic bound.

2) Quadratic distributional distance:

$$
\frac{\int_{-\infty}^{+\infty} \sum_{t=1}^{T}\left|F_{W_{t}\left(z_{(x)}\right)}(u)-F_{W_{t}\left(\min _{i} z_{i}\right)}(u)\right|^{2} d u}{\int_{-\infty}^{+\infty} \sum_{t=1}^{T}\left|F_{W_{t}\left(z_{(x)}\right)}(u)-F_{W_{t}\left(\max _{i} z_{i}\right)}(u)\right|^{2} d u}
$$

This ratio takes into account the quadratic distributional distance between the stochastic bounds and the portfolio.

3) Compound quadratic distance:

$$
\frac{E\left(\sum_{t=1}^{T}\left|W_{t}\left(z_{(x)}\right)-W_{t}\left(\min _{i} z_{i}\right)\right|^{2}\right)}{E\left(\sum_{t=1}^{T}\left|W_{t}\left(z_{(x)}\right)-W_{t}\left(\max _{i} z_{i}\right)\right|^{2}\right)}
$$

Where $W_{t}\left(z_{(x)}\right), W_{t}\left(\max _{i} z_{i}\right)$ and $W_{t}\left(\min _{i} z_{i}\right)$ are the wealth at time $t$ obtained, respectively, from the portfolio $z_{(x)}$, the upper $\left(\max _{i} z_{i}\right)$ and lower $\left(\min _{i} z_{i}\right)$ stochastic bounds. In order to maximize this performance ratio, we should develop the multivariate Markovian evolution of the stochastic bounds and the portfolio following the scheme proposed by Angelelli and Ortobelli (2010). 
Figure 2 shows the ex-post comparison among the three strategies applied on the preselected assets. The blue line shows the results of the absolute distributional distance ratio. The green line represents the results of the quadratic distributional distance, while the red line shows the results obtained using the compound quadratic distance.

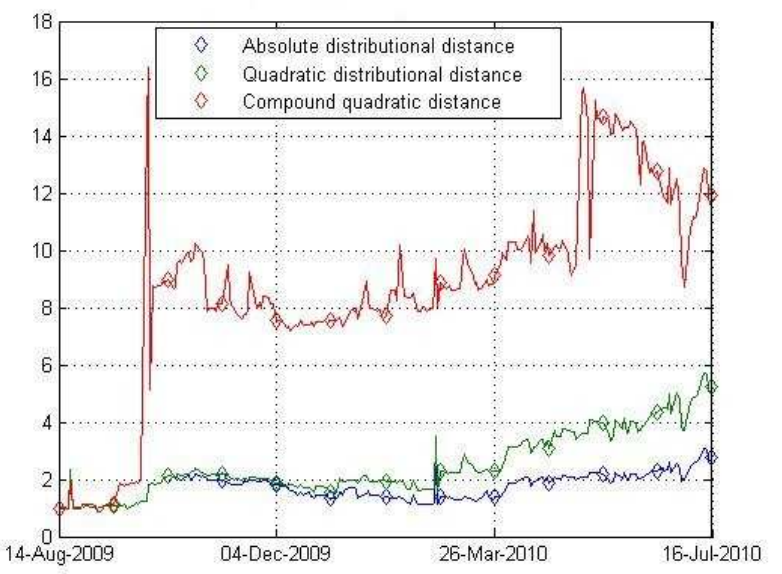

Figure 2. Ex-post final wealth processes comparison (portfolio strategies using the stochastic bounds on the preselected assets).

Some of the preselected assets generate very high returns. These returns could be partially justified by using recent behavioral finance studies on the equity premium puzzle (see Barberis and Huang, 2001, and Benartzi and Thaler, 1995). However, this study is not the main objective of this analysis. The compound quadratic distance shows a very high final wealth (about $1200 \%$ in one year). $97.4 \%$ of the assets chosen with this strategy are traded in Frankfurt, $0.64 \%$ in Taiwan, $0.64 \%$ in Amsterdam, $1 \%$ in Paris, $0.32 \%$ in Brussels. Therefore, most of the assets are traded in Europe. From these results, we deduce that the compound quadratic distance clearly has a greater impact than the distributional distance (see Rachev, 1991). Moreover, all the strategies that use the Markovian hypothesis have a significant impact on choices and, what is fundamental, on the capacity to forecast future evolution of wealth.

\section{Conclusions}

This paper analyzes the impact of Markovianity and pre-selection in optimal portfolio choices. We discussed how to approximate non-parametric Markov processes and showed the impact of both pre-selection and diversification on portfolio problems. Lastly, we propose an empirical comparison among Markovian selection models that consider the distance between upper and lower market stochastic bounds. The expost empirical comparison among different Markovian strategies shows that compound type distances between the stochastic bounds and the portfolio can have a bigger impact on portfolio selection problems.

Moreover, all the results obtained on the global market show dominating results with respect the Sharpe historical approach (see Figure 1).

\section{Acknowledgments}

The authors would like to thank COFIN 60\% 2009 2010 for grants and the seminar audiences at EURO XXIV (Lisbon, 2010).

\section{References}

Angelelli, E. and Ortobelli, S. (2009a). American and European portfolio selection strategies: The Markovian approach. In "Financial Hedging" Nova Science Publishers (eds. P. N. Catlere) 119-152.

Angelelli, E. and Ortobelli, S. (2009b). Maximum Expected Utility of Markovian Predicted Wealth. Lecture Notes in Computer Science 5545, (G. Allen et al. Eds.): ICCS 2009, Part II, LNCS pp. 588-597.

Angelelli, E and Ortobelli, S. (2010). On the application of Markov processes to the large scale portfolio selection problem. Technical Report, University of Brescia.

Barberis, N. and Huang, M. (2001). Mental Accounting, Loss Aversion, and Individual Stock Returns. Journal of Finance. 56(4), 1246-1292.

Benartzi, S. and Thaler, R. H. (1995). Myopic Loss Aversion and the Equity Premium Puzzle. The Quarterly Journal of Economics. 110(1), 73-92.

D'Amico, G. (2003). Markov chain European option: Statistical estimation. Technical Report, Università di Roma "Sapienza". 
Fama, E. (1970). Efficient Capital Markets: A Review of Theory and Empirical Work. Journal of Finance 25 (2), 383-417.

Iaquinta, G. and Ortobelli, S. (2006). Distributional approximation of asset returns with non parametric Markovian trees. International Journal of Computer Science \& Network Security 6(11), 69-74.

Leccadito, A., Ortobelli, S., and Russo, E. (2007). Portfolio selection, VaR and CVaR models with Markov chains. International Journal of Computer Science \& Network Security 7(6), 115-123.

Nelsen, R. B. (2006). An Introduction to Copulas. 2nd ed. Springer.

Ortobelli, S. and Tichy, T. (2010). On the impact of association measures in the portfolio theory. Technical Report, University of Bergamo.

Pflug, G., Wozabal, D., Pichler, A. (2010). Ambiguity and minimaxity in portfolio selection. Presented at EURO XXIV, Lispon.

Rachev, S. (1991). Probability metrics and the stability of stochastic models. Wiley \& Son, Chichester.

Stoyanov, S., Samorodnitsky, G., Rachev, S. T. and Ortobelli, S. (2006). Computing the portfolio Conditional Value-at-Risk in the $\alpha$-stable case. Probability and Mathematical Statistics 26, 1-22.

Tichy, T. and Ortobelli, S. (2009). Concordance Measures and Portfolio Selection Problem. ECON Journal of Economics, Management and Business, 4148. 